\title{
Electromagnetically Induced Transparency in a Double Well Atomic Josephson Junction
}

\author{
J.O. WEATHERALL \\ Department of Logic and Philosophy of Science, University of California Irvine \\ 3151 Social Science Plaza A, Irvine, CA 92697, USA \\ AND C.P. SEARCH \\ Department of Physics and Engineering Physics, Stevens Institute of Technology \\ Castle Point on Hudson, Hoboken, NJ 07030, USA
}

\begin{abstract}
Electromagnetically induced transparency is an important tool for controlling light propagation and nonlinear wave mixing in atomic gases with potential applications ranging from quantum computing to table top tests of general relativity. Here we consider electromagnetically induced transparency in an atomic Bose-Einstein condensate trapped in a double well potential. One well is prepared as in standard electromagnetically induced transparency with a weak probe laser and control laser in a $\Lambda$ configuration while tunneling between the wells provides a coherent coupling between identical electronic states in the two wells leading to the formation of spatially delocalized inter-well dressed states. The macroscopic inter-well coherence of the Bose-Einstein condensate wave function qualitatively modifies the normal electromagnetically induced transparency linear susceptibility and leads to the formation of additional absorption resonances and larger dispersion than electromagnetically induced transparency. We show that these new resonances can be interpreted in terms of the inter-well dressed states and the formation of a novel type of dark state involving the control laser and the inter-well tunneling.
\end{abstract}

PACS numbers: 42.50.Gy, 03.75.Lm

\section{Introduction}

Electromagnetically induced transparency (EIT) [1] is a quantum interference effect that occurs in coherently prepared three-level $\Lambda$ atomic systems. EIT has attracted considerable attention in the past two decades because an ordinarily opaque medium can be made transparent to a probe laser while at the same time the probe experiences dispersion that is tunable over many orders of magnitude along with large third order nonlinear susceptibilities [1]. EIT modifies the optical response of a medium to the probe laser by use of a second laser known as the control that is used to dress the electronic excited state with a third auxiliary level. The weak probe, which normally has only a single excitation path from the ground state to the excited state in the absence of the control beam, now has two excitation pathways to the excited state. These pathways destructively interfere leading to a vanishing absorption at the bare atomic resonance. Along with the vanishing of the probe absorption, the real part of the linear susceptibility, exhibits large normal dispersion leading to extremely slow group velocities [1]. Slow light propagation through EIT systems has been observed experimentally in a variety of media, including hot atomic gases [2] and atomic Bose-Einstein condensates (BEC's) [3], and is now well understood.

Here we describe a modification of the standard 3-level $\Lambda$ EIT configuration that utilizes coherent tunneling of an atomic BEC trapped in a double well potential to further control the optical response for the probe. One well is prepared as a standard EIT system: the electronic excited state is coupled to one of the two ground states via a strong control laser, while a weak probe couples the other ground state to the same excited state. Both lasers are confined to a single well, leaving the second well unperturbed by the lasers. The global phase coherence of the condensate wave function leads to phase coherent tunneling of the condensate wave function between the wells. This tunneling is the origin of the Josephson oscillations of the population difference between the wells, which have recently been observed in a double well condensate [4-6].

This "Josephson junction" of a double well condensate transforms the 3-level $\Lambda$ system of a lone atom into a macroscopic 6-level system spatially distributed between the wells creating four qualitatively new absorption resonances. Two of the resonances result from a splitting of the Autler-Townes absorption resonances characteristic of EIT while the latter two are ultranarrow resonances located in the middle of the EIT transparency window with widths and positions determined by the tunneling frequencies between the wells [7]. We predict that for realistic tunneling rates $(\approx 1 \mathrm{kHz})$, the dispersion to either side of the new resonance can yield group velocities up to two orders of magnitude slower than would be possible in EIT without the tunneling. Although one can in principle reduce the group velocity in EIT by simply turning down the intensity of the control laser, this approach narrows the transparency window and is fundamentally limited by ground state decoherence. By contrast, in a double well 
BEC the ground state decoherence is extremely small with decoherence rates approaching $10-100 \mathrm{~s}^{-1}$.

In Sect. 2, we will outline our model for double well $\Lambda$ BEC dressed by both a control laser and tunnel coupling. In Sect. 3, we derive the system's linear susceptibility, $\chi^{(1)}$. In Sect. 4, we will consider the prospects for experimental observation of these Josephson junction resonances.

\section{Laser dressed Bose condensed Josephson junction}

Let us consider a weakly interacting Bose-Einstein condensate of $N$ atoms confined in a double well potential as depicted in Fig. 1 and recently demonstrated experimentally [4-6]. These experiments represented the first realizations of a single Josephson junction in an atomic BEC. The double well was created in these experiments by superimposing a one-dimensional optical lattice on top of a harmonic optical dipole trap in the $x$-direction. In our model, a necessary assumption is that in the $z$-direction, the harmonic trapping potential is much weaker than in the $x$ or $y$ directions, leading to elongated cigar shaped potentials along the $z$-axis, which maximizes the optical thickness for the probe.

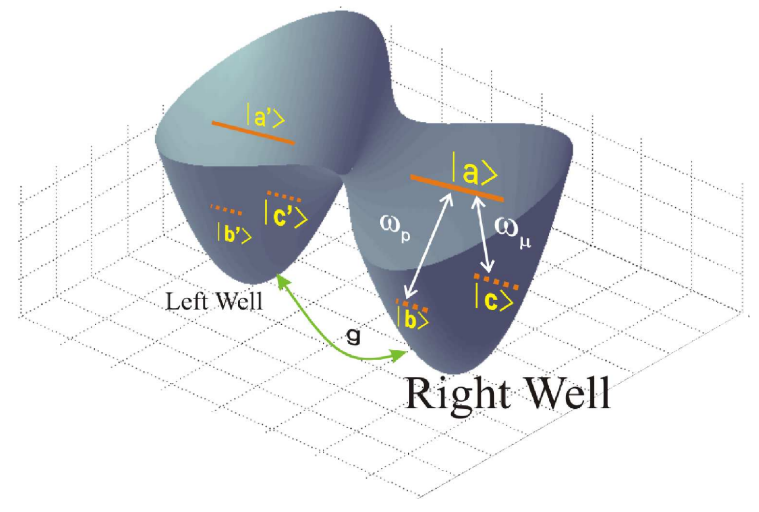

Fig. 1. Schematic description of our system: the atoms in the right well are dressed by a strong control beam near resonance with the $|c\rangle \rightarrow|a\rangle$ transition. We are interested in the behavior of a weak probe beam propagating in the right well, near resonance with the $|b\rangle \rightarrow|a\rangle$ transition. Atoms in electronic state $|\ell\rangle$ are coupled via tunneling through the inter-well barrier to the corresponding states $\left|\ell^{\prime}\right\rangle$ in the left well.

Three internal states of the atoms are coupled by the probe and control laser in a $\Lambda$ configuration and denoted by eigenkets $|a\rangle,|b\rangle$, and $|c\rangle$ where $|a\rangle$ is an electronically excited state, while $|b\rangle$ and $|c\rangle$ are hyperfine ground states of the atoms. The direct transition between the two lower levels is assumed to be dipole forbidden. To distinguish states in the left and right wells we use a to denote the same internal states but in the left well. The right well is dressed with a strong control beam with electric field amplitude $\mathcal{E}_{\mu}$ and frequency $\omega_{\mu}$ that is close to resonance with the energy difference between levels $|a\rangle$ and $|c\rangle$. The propagation through the right well of a weak probe field, $\mathcal{E}_{\mathrm{p}}$, with frequency $\omega_{\mathrm{p}}$ near resonance with the $|b\rangle \rightarrow|a\rangle$ transition is to be studied here. The restriction that the lasers interact with only a single well should be achievable provided the spacing between the wells is sufficiently larger than the diffraction limit given approximately by the wavelength of the light. For example, in the experiment of Ref. [4] the spacing between the wells is $4.4 \mu \mathrm{m}$, which is significantly larger than a typical optical wavelength. Furthermore, the group in Ref. [5] was able to optically resolve a single well to successfully image tunneling dynamics.

We assume that the coupling between wells is weak so that the energy eigenfunctions of the isolated wells serve as a good basis and restrict ourselves to the ground state wave functions, $u^{(\mathrm{L} / \mathrm{R})}(\boldsymbol{r})$, localized in the left $(\mathrm{L})$ or right $(\mathrm{R})$ wells. Furthermore for $T \ll T_{\mathrm{C}}$, excited states of the double well potential are negligible and the condensate is appropriately modeled with a Hartree wave function. In a frame rotating at the control and probe frequencies chosen so that the Hamiltonian is time independent, the Gross-Pitaevskii equations for the probability amplitudes of the six states are

$$
\begin{aligned}
& \mathrm{i} \frac{\partial \tilde{\psi}_{a}}{\partial t}=\left(\omega_{a}-\omega_{\mathrm{p}}-\omega_{\mu}+\tilde{\Psi}^{\dagger} \boldsymbol{U}_{a} \tilde{\Psi}\right) \tilde{\psi}_{a} \\
& \quad-\frac{\Omega_{a b}}{2} \mathrm{e}^{-\mathrm{i} \phi_{a b}} \tilde{\psi}_{b}-\frac{\Omega_{a c}}{2} \mathrm{e}^{-\mathrm{i} \phi_{a c}} \tilde{\psi}_{c}-\frac{g_{a}}{2} \tilde{\psi}_{a^{\prime}}, \\
& \mathrm{i} \frac{\partial \tilde{\psi}_{b}}{\partial t}=\left(\omega_{b}-\omega_{\mu}+\tilde{\Psi}^{\dagger} \boldsymbol{U}_{b} \tilde{\Psi}\right) \tilde{\psi}_{b}-\frac{\Omega_{a b}}{2} \mathrm{e}^{\mathrm{i} \phi_{a b}} \tilde{\psi}_{a}, \\
& \quad-\frac{g_{b}}{2} \tilde{\psi}_{b^{\prime}}, \\
& \mathrm{i} \frac{\partial \tilde{\psi}_{c}}{\partial t}=\left(\omega_{c}-\omega_{\mathrm{p}}+\tilde{\Psi}^{\dagger} \boldsymbol{U}_{c} \tilde{\Psi}^{\prime}\right) \tilde{\psi}_{c}-\frac{\Omega_{a c}}{2} \mathrm{e}^{\mathrm{i} \phi_{a c}} \tilde{\psi}_{a} \\
& \quad-\frac{g_{c}}{2} \tilde{\psi}_{c^{\prime}}, \\
& \mathrm{i} \frac{\partial \tilde{\psi}_{a^{\prime}}}{\partial t}=\left(\omega_{a}-\omega_{\mu}-\omega_{\mathrm{p}}+\tilde{\Psi}^{\prime \dagger} \boldsymbol{U}_{a^{\prime}} \tilde{\Psi}^{\prime}\right) \tilde{\psi}_{a^{\prime}} \\
& -\frac{g_{a}}{2} \tilde{\psi}_{a}, \\
& \mathrm{i} \frac{\partial \tilde{\psi}_{b^{\prime}}}{\partial t}=\left(\omega_{b}-\omega_{\mu}+\tilde{\Psi}^{\prime \dagger} \boldsymbol{U}_{b^{\prime}} \tilde{\Psi}^{\prime}\right) \tilde{\psi}_{b^{\prime}}-\frac{g_{b}}{2} \tilde{\psi}_{b}, \\
& \mathrm{i} \frac{\partial \tilde{\psi}_{c^{\prime}}}{\partial t}=\left(\omega_{c}-\omega_{\mathrm{p}}+\tilde{\Psi}^{\prime \dagger} \boldsymbol{U}_{c^{\prime}} \tilde{\Psi}^{\prime}\right) \tilde{\psi}_{c^{\prime}}-\frac{g_{c}}{2} \tilde{\psi}_{c}
\end{aligned}
$$

with the normalization $\quad \sum_{\ell=a, b, c}\left|\tilde{\psi}_{\ell}(t)\right|^{2}+$ $\sum_{\ell=a^{\prime}, b^{\prime}, c^{\prime}}\left|\tilde{\psi}_{\ell}(t)\right|^{2}=1$. The couplings between levels are moderated by their complex Rabi frequencies defined as $\hbar \Omega_{a c} \mathrm{e}^{-\mathrm{i} \phi_{a c}}=\mathcal{E}_{\mu} D_{a c}$ for the control field and $\hbar \Omega_{a b} \mathrm{e}^{-\mathrm{i} \phi_{a b}}=\mathcal{E}_{\mathrm{p}} D_{a b}$ for the probe field where $D_{i j}=e\langle i|\boldsymbol{x} \cdot \boldsymbol{\epsilon}| j\rangle$ are the dipole moment matrix elements. $\hbar g_{\ell}$ are equal to the Josephson coupling energies, $E_{\mathrm{J}}$, that appear in the Hamiltonian for the bosonic Josephson junction [8, 6] and are proportional to the overlap integral of the wave functions $u_{\ell^{\prime}}^{(\mathrm{L})}(\boldsymbol{r})$ and $u_{\ell}^{(\mathrm{R})}(\boldsymbol{r})$. The two-body interactions are denoted by the 
$3 \times 3$ diagonal matrices $\boldsymbol{U}_{i}$ whose diagonal elements are $\left(\boldsymbol{U}_{i}\right)_{j, j}=\left(4 \pi \hbar a_{i j} N / m\right) \int \mathrm{d}^{3} r\left|u_{i}^{(k)}(\boldsymbol{r})\right|^{2}\left|u_{j}^{(k)}(\boldsymbol{r})\right|^{2}=U_{i j}$ the interaction strengths between states $|i\rangle$ and $|j\rangle$ in the same well in terms of the s-wave scattering length between the two states, $a_{i j}(k=\mathrm{L}$ for primed $i$ and $j$ and $k=\mathrm{R}$ for unprimed indices $i$ and $j$ ). Also, $\tilde{\Psi}=\left(\tilde{\psi}_{a}, \tilde{\psi}_{b}, \tilde{\psi}_{c}\right)^{\mathrm{T}}$ for the right well and with $\tilde{\Psi}^{\prime}$ defined identically for the left well.

To proceed further we must utilize the density matrix $\rho$, defined as the outer product of the probability amplitudes, $\tilde{\psi}_{\ell}$ and calculate the element $\rho_{a b}$ representing the coherence between states $|a\rangle$ and $|b\rangle$. Furthermore, with the density matrix we need to specify decay and decoherence rates for the atoms such that each element of $\rho$ decays as $\dot{\rho}_{i j} \propto-\gamma_{i j} \rho_{i j}$ with $\gamma_{i j}=\left(\gamma_{i}+\gamma_{j}\right) / 2+\tilde{\gamma}_{i j}$ where $\gamma_{i}$ is population decay while $\tilde{\gamma}_{i j}$ is pure dephasing. The excited states $|a\rangle$ and $\left|a^{\prime}\right\rangle$ alone can decay via spontaneous emission at the rate $\gamma_{a}$. Since the population that accumulates in $|a\rangle$ and $\left|a^{\prime}\right\rangle$ is negligibly small due to the weak probe, decay to other levels besides $|b\rangle,\left|b^{\prime}\right\rangle,|c\rangle$, and $\left|c^{\prime}\right\rangle$ is unimportant. We make the following assumption that $\left|\tilde{\gamma}_{a b}-\tilde{\gamma}_{a b^{\prime}}\right| \ll \gamma_{a}$ so that we can set $\gamma_{a b}=\gamma_{a b^{\prime}}=\gamma_{a} / 2+\tilde{\gamma}_{a b}$. This approximation is justified since $\gamma_{a} \approx 10^{7} \mathrm{~s}^{-1}$ while in a condensate $\tilde{\gamma}_{i j} \approx$ 10-1000 s $\mathrm{s}^{-1}$. Additionally, we consider only three types of nonzero decoherence between the ground states, which we allow to vary independently: $\tilde{\gamma}_{b b^{\prime}}, \tilde{\gamma}_{c b}=\tilde{\gamma}_{c b^{\prime}}=\gamma_{\mathrm{C}}$ and $\tilde{\gamma}_{c^{\prime} b}=\tilde{\gamma}_{c^{\prime} b^{\prime}}=\gamma_{C^{\prime}}$.

The equation of motion for $\rho_{a b}$ is

$$
\begin{aligned}
& \mathrm{i} \frac{\partial \rho_{a b}}{\partial t}=\left(\Delta_{\mathrm{p}}-\mathrm{i} \gamma_{a b}+\tilde{\Psi}^{\dagger}\left(\boldsymbol{U}_{a}-\boldsymbol{U}_{b}\right) \tilde{\Psi}\right) \rho_{a b} \\
& \quad+\frac{\Omega_{a b}}{2} \mathrm{e}^{-\mathrm{i} \phi_{a b}}\left(\rho_{a a}-\rho_{b b}\right)-\frac{\Omega_{a c}}{2} \mathrm{e}^{-\mathrm{i} \phi_{a c}} \rho_{c b} \\
& \quad+\frac{g_{b}}{2} \rho_{a b^{\prime}}-\frac{g_{a}}{2} \rho_{a^{\prime} b},
\end{aligned}
$$

where we have defined the probe's detuning from the $|a\rangle \rightarrow|b\rangle$ transition, $\Delta_{\mathrm{p}}=\omega_{a}-\omega_{b}-\omega_{\mathrm{p}}$. Since we are solving to linear order in the probe under the assumption $\Omega_{\mathrm{p}} \ll \gamma_{a b}, \rho_{a a}, \rho_{a^{\prime} a^{\prime}}, \rho_{c c}, \rho_{c^{\prime} c^{\prime}}, \rho_{c c^{\prime}}, \rho_{a c}, \rho_{a c^{\prime}}, \rho_{a^{\prime} c}, \rho_{a^{\prime} c^{\prime}}$, $\rho_{a^{\prime} b}, \rho_{a^{\prime} b^{\prime}} \approx 0$ since they are all of order $\left(\Omega_{\mathrm{p}} / \gamma_{a b}\right)^{2}$ or higher in perturbation theory.

In order to keep the inter-well couplings to all orders, we move to a partially dressed state basis, in which the $\left\{|b\rangle,\left|b^{\prime}\right\rangle\right\}$ and $\left\{|c\rangle,\left|c^{\prime}\right\rangle\right\}$ subspaces of our Hamiltonian are diagonalized. Applying this transformation produces dressed states $|B\rangle=\cos \theta_{b}|b\rangle+\sin \theta_{b}\left|b^{\prime}\right\rangle$ and $\left|B^{\prime}\right\rangle=-\sin \theta_{b}|b\rangle+\cos \theta_{b}\left|b^{\prime}\right\rangle$ for $\left\{|b\rangle,\left|b^{\prime}\right\rangle\right\}$ and $|C\rangle=$ $\cos \theta_{c}|c\rangle+\sin \theta_{c}\left|c^{\prime}\right\rangle$ and $\left|C^{\prime}\right\rangle=-\sin \theta_{c}|c\rangle+\cos \theta_{c}\left|c^{\prime}\right\rangle$ for $\left\{|c\rangle,\left|c^{\prime}\right\rangle\right\}$. In the diagonalized bases, the energy eigenvalues are $E_{b / c}^{( \pm)}= \pm \hbar \Omega_{b / c}^{\text {eff }} / 2$ for the two subspaces. Here $\cos \theta_{b / c}=\left[\left(1+\Delta_{b / c} / \Omega_{b / c}^{\text {eff }}\right) / 2\right]^{1 / 2}, \sin \theta_{b / c}=[(1-$ $\left.\left.\Delta_{b / c} / \Omega_{b / c}^{\mathrm{eff}}\right) / 2\right]^{1 / 2}$, and $\Omega_{b / c}^{\mathrm{eff}}=\sqrt{\Delta_{b / c}^{2}+g_{b / c}^{2}}$. The nonlinear detunings are $\Delta_{b / c}=\tilde{\Psi}^{\dagger} \boldsymbol{U}_{b / c} \tilde{\Psi}-\tilde{\Psi}^{\prime \dagger} \boldsymbol{U}_{b^{\prime} / c^{\prime}} \tilde{\Psi}^{\prime}$. In terms of these dressed states, the coherence $\rho_{a b}$ is given by $\rho_{a b}=\cos \theta_{b} \rho_{a B}-\sin \theta_{b} \rho_{a B^{\prime}}$. Using these dressed state transformations in the remaining density matrix equa- tions, we find that the density matrix equations simplify in two sets of three equations that are uncoupled from each other that can be solved analytically for $\rho_{a B}$ and $\rho_{a B^{\prime}}$.

\section{Affect of Josephson tunneling on probe susceptibility}

The polarization for the probe is related to $\rho_{a b}$ by $\mathcal{P}=2 N\left[u_{a}(\boldsymbol{r})\right]^{*} u_{b}(\boldsymbol{r}) D_{a b} \rho_{a b}$. Likewise, the complex linear susceptibility is given by $\chi^{(1)}=\mathcal{P} /\left(\epsilon_{0} \mathcal{E}_{\mathrm{p}}\right)$, which determines both the absorption coefficient, $\alpha\left(\omega_{\mathrm{p}}\right)=$ $k_{\mathrm{p}} \Im\left[\chi^{(1)}\right]$ and the index of refraction, $n\left(\omega_{\mathrm{p}}\right) \approx(1+$ $\left.\Re\left[\chi^{(1)}\right]\right)^{1 / 2}$. The spatial term $\left[u_{a}(\boldsymbol{r})\right]^{*} u_{b}(\boldsymbol{r})$ reflects the density profile of the atoms and only determines the optical thickness of the condensate. In our analysis we will focus on the reduced susceptibility $\tilde{\chi}^{(1)}=$ $\epsilon_{0} \hbar \gamma_{a b} \chi^{(1)} /\left(2 D_{a b}^{2} N\left[u_{a}(\boldsymbol{r})\right]^{*} u_{b}(\boldsymbol{r})\right)=\gamma_{a b} \rho_{a b} / \Omega_{\mathrm{p}}$.

The simplest case to consider is when the dressed state mixing angles are $\theta_{b}=\theta_{c}=\pi / 4$ when the solution simplifies considerably and is given by

$$
\begin{aligned}
& \tilde{\chi}^{(1)}=\frac{\gamma_{a b}}{2}\left\{\left[\left(\Delta_{\mu}-\Delta_{\mathrm{p}}+\mathrm{i} \gamma_{\mathrm{C}^{\prime}}-\Omega_{b} / 2\right)\right.\right. \\
& \left.\quad \times\left(\Delta_{\mu}-\Delta_{\mathrm{p}}+\mathrm{i} \gamma_{\mathrm{C}}-\Omega_{b} / 2\right)-\Omega_{c}^{2} / 4\right] / Z_{1} \\
& \quad+\left[\left(\Delta_{\mu}-\Delta_{\mathrm{p}}+\mathrm{i} \gamma_{\mathrm{C}^{\prime}}+\Omega_{b} / 2\right)\right. \\
& \left.\left.\quad \times\left(\Delta_{\mu}-\Delta_{\mathrm{p}}+\mathrm{i} \gamma_{\mathrm{C}}+\Omega_{b} / 2\right)-\Omega_{c}^{2} / 4\right] / Z_{2}\right\},
\end{aligned}
$$

where $Z_{1}=\left(\Delta_{\mathrm{p}}-\mathrm{i} \gamma_{a b}+\Omega_{b} / 2\right)\left[\left(\Delta_{\mu}-\Delta_{\mathrm{p}}+\mathrm{i} \gamma_{\mathrm{C}^{\prime}}-\right.\right.$ $\left.\left.\Omega_{b} / 2\right)\left(\Delta_{\mu}-\Delta_{\mathrm{p}}+\mathrm{i} \gamma_{\mathrm{C}}-\Omega_{b} / 2\right)-\Omega_{c}^{2} / 4\right]+\left(\Delta_{\mu}-\Delta_{\mathrm{p}}+\mathrm{i} \gamma_{\mathrm{C}^{\prime}}-\right.$ $\left.\Omega_{b} / 2\right) \Omega_{\mu}^{2} / 4$ and $Z_{2}=\left(\Delta_{\mathrm{p}}-\mathrm{i} \gamma_{a b}-\Omega_{b} / 2\right)\left[\left(\Delta_{\mu}-\Delta_{\mathrm{p}}+\mathrm{i} \gamma_{\mathrm{C}^{\prime}}+\right.\right.$ $\left.\left.\Omega_{b} / 2\right)\left(\Delta_{\mu}-\Delta_{\mathrm{p}}+\mathrm{i} \gamma_{\mathrm{C}}+\Omega_{b} / 2\right)-\Omega_{c}^{2} / 4\right]+\left(\Delta_{\mu}-\Delta_{\mathrm{p}}+\mathrm{i} \gamma_{\mathrm{C}^{\prime}}+\right.$ $\left.\Omega_{b} / 2\right) \Omega_{\mu}^{2} / 4$. We have redefined $\Delta_{\mathrm{p}}=\omega_{a}-\omega_{b}-\omega_{\mathrm{p}}+U_{a b}$ and $\Delta_{\mu}=\omega_{a}-\omega_{c}-\omega_{\mu}+U_{a b}$ as the probe and control beam detunings, respectively. It is worth pointing out that in the limit that $\Omega_{b}, \Omega_{c} \rightarrow 0$, we recover from Eq. (3) the standard expression for the EIT linear susceptibility in a $\Lambda$ system.

Atomic spontaneous emission rates for atoms commonly used in BEC experiments (Na, Rb, Li) are typically on the order of $10 \mathrm{MHz}$. Typical tunneling times were on the order of $10 \mathrm{~ms}$ in Ref. [4] while more recent experiments achieved tunnel couplings between the wells as high $7900 \mathrm{~Hz}$ [6] implying $g_{\ell} \ll \Omega_{a c}, \gamma_{a}$. An example of the real and imaginary parts of the susceptibility is shown in Fig. 2. We see that the presence of the second well manifests itself as two ultranarrow resonances located inside of the EIT transparency window. When $\Delta_{b}=0$, the new resonances are symmetrically located about $\Delta_{\mathrm{p}}=0$ at the locations $\pm g_{b} / 2$. In general the location of these resonances is $\Delta_{\mathrm{p}}= \pm \Omega_{b}^{\text {eff }} / 2$ and for $\Omega_{a c}, \gamma_{a b} \gg g_{b}, g_{c}$ their shape is approximately Lorentzian

$$
\begin{aligned}
& \Im\left[\tilde{\chi}^{(1)}\right] \approx \frac{\gamma_{a b} g_{c}^{2}}{2 \Omega_{\mu}} \\
& \quad \times\left\{\frac{g_{c}^{2} / \Omega_{\mu}^{2}+\gamma_{\mathrm{C}^{\prime}} / \gamma_{a b}}{\left(\Delta_{\mathrm{p}} \mp g_{b} / 2\right)^{2}+\left[\gamma_{a b}\left(g_{c}^{2} / \Omega_{\mu}^{2}+\gamma_{\mathrm{C}^{\prime}} / \gamma_{a b}\right)\right]^{2}}\right\}
\end{aligned}
$$


It is the sum of the "power broadening" term $2 \gamma_{a b} g_{c}^{2} / \Omega_{\mu}^{2}$ and the dephasing rate for $\left|c^{\prime}\right\rangle$. Similar results have been obtained by Lukin et al. [9] and Mahmoudi et al. [10] who studied EIT in 4-level system. Off-resonance these lines fall off much faster than a Lorentzian and in the limit of vanishing decoherence, there are absorption nulls located on either side of these resonances.

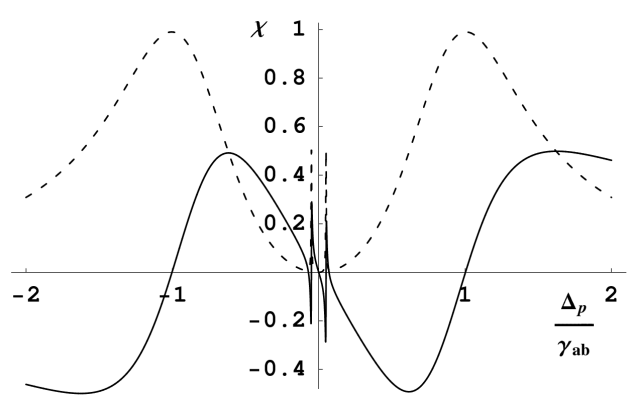

Fig. 2. The full probe spectrum for the $|a\rangle-|b\rangle$ transition. The $\Im\left[\chi^{(1)}\right]$ is plotted as a dotted line, and $\Re\left[\chi^{(1)}\right]$ as a solid line. Let us note the two additional features, symmetrically located around zero detuning, at $\pm g_{b} / 2$. In this plot we have taken $g_{b}=g_{c}=\gamma_{a b} / 10(\approx 500 \mathrm{kHz})$ to emphasize the modifications to the standard EIT spectrum. Given more physically realistic parameters, the features would be considerably narrower and closer together. Let us note that here $\Omega_{a c}=\gamma_{a}=2 \gamma_{a b}$ and $\gamma_{\mathrm{C}}=\gamma_{\mathrm{C}}^{\prime}=0$.

These new resonances can be understood in terms of the interaction of the dressed states of the $|b\rangle$ and $\left|b^{\prime}\right\rangle$ subsystem with the eigenstates of the $\left|c^{\prime}\right\rangle \leftrightarrow|c\rangle \leftrightarrow|a\rangle$ subsystem. The energies of $|B\rangle$ and $\left|B^{\prime}\right\rangle$ are $\hbar \omega_{B, B^{\prime}}=\hbar \omega_{b}+$ $U_{b b} \pm \Omega_{b}^{\text {eff }} / 2$. Therefore even in the absence of the control laser, the $|b\rangle \rightarrow|a\rangle$ absorption line would be split into two new lines. The $\left|c^{\prime}\right\rangle \leftrightarrow|c\rangle \leftrightarrow|a\rangle$ subspace is a three-level system that is isomorphic to a $\Lambda$ atom. Again assuming $\Delta_{c}=0$ and the control laser is on resonance, $\Delta_{\mu}=0$, then the Hamiltonian for the $\left\{|a\rangle,|c\rangle,\left|c^{\prime}\right\rangle\right\}$ subsystem is $\boldsymbol{H}=\hbar \omega_{a} \boldsymbol{I}+(\hbar / 2)\left(\Omega_{a c}|a\rangle\left\langle c\left|-g_{c}\right| c\right\rangle\left\langle c^{\prime}\right|+\right.$ h.c. $)$. The eigenstates of this Hamiltonian are $\left|a_{+}\right\rangle=\frac{1}{\sqrt{2}}(\sin \theta|a\rangle+$ $\left.|c\rangle+\cos \theta\left|c^{\prime}\right\rangle\right),\left|a_{-}\right\rangle=\frac{1}{\sqrt{2}}\left(\sin \theta|a\rangle-|c\rangle+\cos \theta\left|c^{\prime}\right\rangle\right)$, and $\left|a_{0}\right\rangle=\cos \theta|a\rangle-\sin \theta\left|c^{\prime}\right\rangle$ where $\tan \theta=-\Omega_{a c} / g_{c}$. The energies of the states $\left|a_{ \pm}\right\rangle$are $E_{ \pm}=\hbar \omega_{a} \pm \hbar \sqrt{\Omega_{a c}^{2}+g_{c}^{2}} / 2$ while $\left|a_{0}\right\rangle$ has energy $E_{0}=\hbar \omega_{a}$. As one can see, $\left|a_{0}\right\rangle$ is the same type of dark state that appears in stimulated Raman adiabatic passage (STIRAP) and coherent population trapping. In this case, this tunneling induced dark state is a superposition of $|a\rangle$ and $\left|c^{\prime}\right\rangle$ but not $|c\rangle$. Transitions from the $\left\{|B\rangle,\left|B^{\prime}\right\rangle\right\}$ manifold to $\left|a_{0}\right\rangle$ will then exhibit absorption resonances at $\omega_{a}-\omega_{B, B^{\prime}}$, which correspond to the new ultranarrow features. All in all there are six transitions that should appear as resonances in the absorption spectrum corresponding to transitions from $\left\{|B\rangle,\left|B^{\prime}\right\rangle\right\}$ dressed manifold to $\left\{\left|a_{+}\right\rangle,\left|a_{-}\right\rangle,\left|a_{0}\right\rangle\right\}$ states via the probe [11]. The transitions to the two "bright" states $\left|a_{ \pm}\right\rangle$correspond to the main absorption peaks lo- cated at $\Delta_{\mathrm{p}} \approx \pm \Omega_{a c} / 2$ for $\Omega_{a c} \gg g_{c}, g_{b}$. Each of these resonances actually consist of a pair of resonances separated by a distance $g_{b}$ but because $\gamma_{a b} \gg g_{b}$ these pairs cannot be individually resolved.

As one can see from Eq. (3) there is a transparency window of width $g_{b}$ in between the two ultranarrow resonances with $\Im\left[\chi^{(1)}\right]=0$ at $\Delta_{\mathrm{p}}=0$. To either side of these resonances, the absorption is negligible. In the vicinity of these resonances the dispersion, $\partial \Re\left[\chi^{(1)}\right] / \partial \omega_{\mathrm{p}}$, is extremely large and, to either side of the peak, there is a region of width $\approx g_{c} / 2$ in which the dispersion is 10 times greater than in standard EIT while within a region of width $\approx g_{c} / 8$, the dispersion is amplified by a factor of 100. (Let us note that "standard EIT" refers to the case where $g_{a}=g_{b}=g_{c}=0$ but with all other parameters being the same.) The absorption, meanwhile, drops to below $1 \%$ within an order of magnitude of the feature's width $-2\left(g_{c} / \Omega_{a c}\right)^{2} \gamma_{a b}$ - from the center of the resonance, and is negligible within the regions of interest. Using $g_{c} \approx 1 \mathrm{kHz}$, we find there is a region, to either side of the peaks, of width $\mathcal{O}(1 \mathrm{kHz})$ in which the absorption is negligible $(\lesssim 0.001 \%)$ and the dispersion is $\approx 10$ times greater than in standard EIT with a control laser of the same strength; similarly there is a region of width $\mathcal{O}(100 \mathrm{~Hz})$ in which the dispersion is $\approx 100$ times greater than standard EIT, again with negligible absorption (likewise $\lesssim 0.001 \%$ ). This implies that the group velocity $[1,3], v_{\mathrm{g}}\left(\omega_{\mathrm{p}}\right)=c /\left[n+\left(\omega_{\mathrm{p}} / 2 n\right)\left(\partial \Re\left[\chi^{(1)}\right] / \partial \omega_{\mathrm{p}}\right)\right]$ could be made significantly smaller than in previous experiments. Let us note that the slope of the dispersion is rapidly changing within each of these regions, and so any pulse transmitted through the well would undergo considerable reshaping.

\section{Conclusions}

The ultranarrow resonances and regions in which the dispersion is especially high are on the order of $g_{c}$ or smaller, begging the question of their experimental accessibility using readily available equipment. One solution follows a recent paper by Pfau's group, at Stuttgart [12]. In their experiment, only a $\sigma^{+}$polarized probe couples the initial state of the atoms to the same excited state as the control laser. On the other hand, a $\sigma^{-}$polarized probe couples off-resonantly to other excited states that are unaffected by the control laser. By mixing a small amount of $\sigma^{-}$polarized light into their otherwise $\sigma^{+}$polarized probe beam, they simultaneously measured the absorption and dispersion of the EIT system by examining the interference pattern between the $\sigma^{+}$and $\sigma^{-}$ polarized components of the probe. They report observing features as narrow as $4 \mathrm{kHz}$ in the $\Re\left[\chi^{(1)}\right]$. A similar experiment with a system prepared as described in this paper would test our predictions for the modified features in $\chi^{(1)}$ arising from interwell tunneling.

In conclusion we have studied EIT in an atomic Bose condensed Josephson junction and the affect of phase coherent tunneling of the condensate wave function on the 
susceptibility of a probe laser in one well. The Josephson coupling leads to the appearance of four new absorption resonances in the probe susceptibility and two of these resonances, which are located inside the EIT transparency window, have widths limited only by ground state dephasing and the tunneling frequency. These new narrow resonances dramatically modify the dispersion leading to significantly slower group velocities in regions of negligible absorption.

\section{References}

[1] M. Fleischhauer, A. Imamoglu, J.P. Marangos, Rev. Mod. Phys. 77, 633 (2005).

[2] M.M. Kash, V.A. Sautenkov, A.S. Zibrov, L. Holberg, G.R. Welch, M.D. Lukin, Y. Rostovtsev, E.S. Fry, M.O. Scully, Phys. Rev. Lett. 82, 5229 (1999).

[3] L.V. Hau, S.E. Harris, Z. Dutton, C.H. Behroozi, Nature 397, 594 (1999).
[4] M. Albiez, R. Gati, J. Fölling, S. Hunsmann, M. Cristiani, M. Oberthaler, Phys. Rev. Lett. 95, 010402 (2005).

[5] S. Levy, E. Lahoud, I. Shomroni, J. Steinhauer, Nature 449, 579 (2007).

[6] R. Gati, B. Hemmerling, J. Folling, M. Albiez, M.K. Oberthaler, Phys. Rev. Lett. 96, 130404 (2006).

[7] J.O. Weatherall, C.P. Search, M. Jääskeläinen, Phys. Rev. A 78, 013830 (2008).

[8] D. Ananikian, T. Bergeman, Phys. Rev. A 73, 013604 (2006).

[9] M.D. Lukin, S.F. Yelin, M. Fleischhauer, M.O. Scully, Phys. Rev. A 60, 3225 (1999).

[10] M. Mahmoudi, R. Fleischhaker, M. Sahrai, J. Evers, J. Phys. B, At. Mol. Opt. Phys. 41, 025504 (2008).

[11] J.O. Weatherall, C.P. Search, Phys. Rev. A 78, 053802 (2008).

[12] B. Kaltenhäuser, H. Kübler, A. Chromik, J. Stuhler, T. Pfau, A. Imamoglu, J. Phys. B, At. Mol. Opt. Phys. 40, 1907 (2007). 